\title{
O Papel do Entendimento na Aceitação de Teorias Científicas
}

\author{
The Role of Understanding in Accepting \\ Scientific Theories
}

\author{
Breno Pascal de Lacerda Brito \\ Doutor em Ensino, Filosofia e História das Ciências na UFBA \\ Pesquisador do Instituto Nacional de Ciência e Tecno- \\ logia em Estudos Interdisciplnares e Transdisciplinares \\ em Ecologia e Evolução (INCT IN-TREE)
}

Resumo: O critério de aceitação de uma teoria científica deve ser pautado no Entendimento da mesma. Isso porque a concepção de aceitação de teorias científicas com base na expressão dos valores epistêmicos não é possível, dado o problema da incomensurabilidade. Para resolver essa questão devemos considerar as competências de um agente epistêmico e como as mesmas sustentam sua aceitação. Ao analisar o processos de escolha de teorias científica, se pode concluir que o mesmo não está associado a mera formação de crenças em sua adequação empírica. Sendo um processo não vinculado com a verdade, e sim associado com a melhor explicação, consequentemente gerando melhor Entendimento, o qual é, logicamente e conceitualmente, quesito necessário para Aceitação de dado estado cognitivo consequente de uma Explicação. Como o Entendimento é uma característica de um agente virtuoso, podemos crer que a teoria escolhida seria resultado da habilidade de um agente virtuoso, e consequentemente uma teoria confiável. 
Palavras-Chave: Virtude Epistêmica; Agente Epistêmico; Verdade; Propositivo; Não-Propositivo.

Abstract: The acceptance criterion of a scientific theory must be guided on its Understanding. That is so because the acceptance conception of scientific theories based on the expression of epistemic values is not possible, given the incommensurability issue. To solve this matter we must consider the epistemic agent competences and how these sustain its acceptance. In analyzing the choosing process of scientific theories, one must conclude that it is not associated with the mere belief formation on its empirical adequation. Thus being a process not bound to the truth, but associated with the best explanation, therefore generating the best Understanding, which is logically and conceptually, necessary aspect on acceptance of a given cognitive state consequent of an Explanation. Since the Understanding is a characteristic of a virtuous agent, we must believe that the chosen theory would be a result of the ability of a virtuous agent, and therefore a reliable theory.

Keywords: Epistemic Virtue; Epistemic Agent; Truth; Propositional; Non-Propositional.

$\mathbf{U}^{n}$ ma das principais questões para a filosofia das ciências, desde o início do século XX, é justamente qual o procedimento deve ser adotado para a escolha das teorias. Essa questão é agravada quando se tem em vista a escolha de teorias empiricamente equivalentes, ou seja, teorias rivais que têm o mesmo poder de explicar seu conjunto de dados (sendo os mesmos conjuntos de dados, ou não). Ao considerar a falência do programa da demarcação (tanto pelo Confirmacionismo quanto pelo Falseabilismo), a busca por um método algoritmo para essa empreitada perdeu forças e os filósofos da ciência se voltaram então para outras propostas que poderiam trazer uma resposta a esse problema (Godfrey-Smith, 2003).

Atualmente, a principal resposta para esse problema envolve analisar o conjunto de valores epistêmicos associados a cada teoria, como propõe Kuhn (1998), van Fraassen (2006), Lacey (1999), entre outros. Porém, apesar de supostamente essa ser uma solução para o problema ela em si acarreta uma 
nova gama de problemas associados. O maior dele é quais os valores que devem ser analisados, como analisa-los (Ivanova, 2014) e quais critérios epistêmicos que devem ser adotados na análise (Elgin, 2013).

Hugh Lacey (1999), filósofo e sociólogo das ciências, pretendendo resolver esse problema apresenta então um modelo descritivo-normativo da prática científica, propondo como os cientistas podem resolver o problema de escolha de teorias e manter sua objetividade. Ele propõe que o fazer ciência envolve três fases distintas: a primeira seria a da escolha da estratégia de pesquisa, envolvendo valores epistêmicos e valores não-epistêmicos. A segunda seria da aceitação da teoria, envolvendo apenas valores epistêmicos, e por fim, a terceira seria do uso da teoria, envolvendo assim valores epistêmicos e não-epistêmicos novamente. Tendo em vista esse modelo, o segundo momento então seria o mais relevante e aquele que trataria da escolha da teoria per se. A proposta de Lacey se encontra em consonância com outros autores ao considerar apenas os valores epistêmicos da teoria para sua aceitação.

Porém, ao considerar as particularidades do processo de aceitação epistêmica, não há uma concordância em quais seus critérios fundamentais com o que Lacey (e outros filósofos) determinam. Isso porque a aceitação não pode ser reduzida a crença da adequação empírica, sendo um processo diferente e que pressupõe um nível epistêmico de reflexão superior ao da crença (mesmo racional) (Lehrer, 2000). Assim o processo de aceitação estaria associado não a análise no nível da teoria em si, e sim ao nível da reflexão e consideração do agente epistêmico. Essa mudança de nível de consideração não só se deve dar para melhor podermos explicar como ocorre o processo da aceitação, mas também por que pautar o mesmo ao nível da teoria e seus valores associados não parece ser possível (Ivanova, 2014).

Dessa forma a pergunta que deve ser feita é como analisar o caso a partir do papel que o conhecimento ou o entendimento desempenhariam no processo de aceitação. Para responder essa colocação, tentarei defender que é o Entendimento e não o Conhecimento que pauta a aceitação das teorias científi- 
cas. Isso porque é o entendimento que melhor reflete a capacidade de explicação de uma teoria (Riggs, 2007), assim como ao focar no mesmo podemos evitar problemas fundamentais que se apresentam ao se apelar a uma noção comumente discutida na epistemologia sobre conhecimento, como os problemas associados a justificação, respostas a casos tipo Gettier e respostas ao cético (Zagzebski, 2001). Para tanto, primeiro devo apontar o porquê o nível da análise ao nível da teoria não é possível se formos nos pautar sobre os valores epistêmicos da própria teoria e sim deve ser feito ao nível do agente epistêmico.

Consideremos duas teorias concorrentes, A e B. Ambas são empiricamente adequadas. Assim a proposta para determinar sua aceitação seria de nos debruçássemos sobre seus valores epistêmicos para então selecionar qual seria a teoria aceita. Dessa forma, o nível de análise seria a própria teoria e quão bem ela apresenta uma lista de valores previamente aceitos como necessários a sua escolha (Lacey 1999).

Contudo ao analisar esse tipo de lista de valores associados a teorias, há uma variação sobre quais valores devem ser incluídos e seus pesos, que pouco é capaz de informar qual melhor teoria (Elgin, 2013). Também há uma variação sobre o que cada valor associado a teoria realmente quer dizer e como eles devem ser analisados. Ivanova (2014) apresenta uma defesa justamente da não possibilidade de se escolher teorias rivais com base meramente dos valores internos das mesmas (e.g. consideremos o valor epistêmico da simplicidade da teoria: como devemos analisá-lo? Por quantidade de pressupostos matemáticos? Por quantidade de variáveis? Por comprometimento ontológico?). A autora defende que o nível de consideração para a aceitação da teoria não é a própria teoria como defendem os filósofos da ciência, e sim a motivação do cientista. É ele, enquanto agente epistêmico, que irá tomar a decisão de qual teoria aceitar e qual o critério se deve ter para pautar a aceitação.

Essa decisão de analisar a escolha de teorias a partir do agente é similar ao giro de análise que a epistemologia das virtudes propõe. Ao não encontrar respostas satisfatórias para o desafio cético, e consequentemente, não conseguir respon- 
der o porquê dada justificativa garante um maior valor ao conhecimento, os epistemologos defendem que a análise deve ser outra (Pritchard, 2007). Essa análise deve focar no Agente Epistêmico Virtuoso, dado que habilidade seria o que garantiria o valor ao conhecimento gerado (Zagzebski, 2001). E esse conhecimento com maior valor seria o aceito.

Ao tratar sobre a aceitação, alguns autores defendem que há uma sobreposição desta com a crença verdadeira, sendo assim, a aceitação estaria pautada no caráter de verdade da crença (Engel, 2000). Ou seja, o objetivo de se aceitar dada teoria seria dado a crença que ela possa acessar a verdade (ou ser mais próxima da verdade). Porém há pontos fundamentais que devem ser explorados para levamos em consideração o papel da crença verdadeira no processo de aceitação, sendo eles:

1. Não há necessariamente uma sobreposição da crença e da aceitação;

2. O papel da aceitação da teoria cientifica não deve ser pautada pela verdade;

3. A aceitação deve estar relacionada com o papel da explicação nas ciências;

1. O primeiro ponto versa sobre o que uma literatura vem discutindo nos últimos anos, tendo um de seus marcos a obra de Cohen (1992). Cohen defende que crença e aceitação são dois estados cognitivos diferentes, e apesar deles poderem ser consoantes eles não são mutuamente necessários. Ou seja, é possível que se aceite um enunciado sem acreditar nele e vice e versa. Com base nessa postura se defende que o marco diferenciador entre eles é que a crença seria um caráter majoritariamente involuntário, caracterizado sobre um estado cognitivo de primeira ordem, enquanto aceitação seria uma atitude consciente, caracterizado por um estado metacognitivo e de segunda ordem (Lehrer, 1999). Outro ponto é que a aceitação suporta que se possa aceitar algo que não seja verdadeiro, como por exemplo, aceitar que dado político seja corrupto, mesmo não sendo verdadeiro (Cohen, 2000; Sainsbury, 1970). Ou seja, ao aceitar dado enunciado o compromisso epistêmico é apenas de trata-lo enquanto verdade para as derivações desse enunciado (Cohen, 1992). 
Por sua vez, Lehrer (2000) defende que a crença estaria relacionada apenas com a informação não necessariamente refletida, enquanto a aceitação seria relacionada com uma reflexão sobre a informação, sendo assim associada ao Conhecimento, pois aceitar algo é apresentar a justificativa para a informação/crença em questão. Porém, a divisão de Lehrer, apesar de útil e capturar uma importante característica da aceitação (ser fruto de uma reflexão cognitiva sobre dado objeto), não consegue explicar casos onde a aceitação de um dado evento não é reduzida ao conhecimento, como em casos que se aceita algo resultante da sorte epistêmica, ou quando se aceita algo não propositivo. Imaginemos o caso onde eu desafio um campeão de dardos para uma competição. Porém, por acaso, no dia da competição o campeão esteja com uma leve infecção auditiva que influencie seu equilíbrio o fazendo errar a maioria dos arremessos, resultando assim na minha vitória. Desta forma se pode aceitar que eu tenha jogado melhor que o campeão nessa ocasião, mas isso não foi dado minhas capacidades e sim porque eu tive sorte. Outro caso é que eu possa aceitar o funcionamento de um dado automóvel a partir da relação das partes de seu motor, sem necessariamente reduzir essa relação a uma questão de proposições. Essa análise está fortemente associada com problemas clássicos sobre o Conhecimento, ainda mais de uma perspectiva confiabilista do mesmo e a necessidade de resposta ao cético com base na verdade. Ao apelarmos para a aceitação, de forma que ela possa ser não propositiva e não tem como fim a verdade, superamos esse problema do conhecimento e da necessidade da crença.

2. Ao pautar a teoria cientifica na verdade negamos o papel não dogmático da mesma, influenciada por questões para além da adequação empírica, e mesmo as críticas de como se pode analisar a verdade dos testes científicos, sua história (e.g. muitas das teorias mais aceitas da ciência ao longo de sua história não são mais aceitas hoje, ou não refletem o mundo como ele é) e a própria prática científica. A própria ciência para dar certo muitas vezes propõe teorias que não se propõe verdadeiras para que possa explicar fenômenos isolados (como a física clássica ignorar atrito, ou o mendelismo se propor a um sistema simples 
e irreal da regulação gênica) como defende Cohen (1992, 2000). Uma resposta a essa alegação pode ser a defesa que a crença não é sobre a teoria em si e sim sobre a capacidade explicativa que uma teoria científica possa ter. Intuitivamente não me parece que esse deslocamento da crença consiga mudar a relação, isso porque o nível de análise primário é sobre uma teoria especificada qualquer e sua relação com o mundo e não sobre capacidades gerais de teorias cientificas explicarem fenômenos. Se esse fosse o caso o cientista acreditaria em toda e qualquer teoria cientifica que tivesse o mínimo de relevância empírica e aceitar suas derivações, o que não me parece verossímil. Outro ponto que ao apelar para a crença na capacidade explicativa se está analisando não só a questão sobre uma ótica onde a crença é voluntária (no momento que se depende da amplitude da capacidade de explicação para se crer num enunciado) e contexto dependente, o que desclassifica enquanto crença por definição. Ainda se pode mostrar a fragilidade de argumento de crença deslocada, ao considerar que a explicação de uma teoria pode ir além de critérios proposicionais (como o uso de gráficos, experimentos, etc) minando assim sua associação com conhecimento, que tem sua base na crença verdadeira proposicional (Ferreira, 2015; Zagzebski, 2001). Mesmo que aceitemos que a ciência não busca teorias reais e apenas teorias confiáveis sobre o mundo, adotando assim uma perspectiva confiabilista do conhecimento, essa perspectiva iria encontrar o problema de que apenas a postura confiabilista não consegue lidar com a questão da sorte epistêmica, nem com o problema da troca.

3. Acredito que esse seja o ponto mais importante dos elencados até agora, dado que está diretamente associado com o papel das ciências. De forma deflacionada podemos aceitar que a ciência tem como objetivo explicar os fenômenos naturais e sociais de seu escopo, devemos então considerar que o processo de aceitação está diretamente associado ao poder explicativo da teoria em análise. Aqui estaria o principal motivo de associar a aceitação ao entendimento e não ao conhecimento. Isso porquê explicar dada teoria é apresentar a coerência interna das relações das partes envolvidas do fenômenos para que se possa então compreender como o mesmo funciona como um todo 
(Riggs, 2007; Ferreira, 2015), principalmente ao considerarmos que muitos dos fenômenos detêm um caráter sinergético, impossibilitando assim sua redução a soma de suas partes e assim meramente a uma relação direta de causa e efeito. Como defende Riggs (2007), essa é a função do Entendimento e onde repousa seu valor intrínseco, a compreensão das partes constituintes de dado fenômeno em um todo coerente. O conhecimento por sua vez não necessariamente resulta em entendimento, ou seja, a compreensão coerente de todo o sistema ${ }^{48}$ (Zagzebski, 2001). Um exemplo disso é o modelo da dupla hélice do $\mathrm{ADN}$, onde Watson e Crick a partir da foto da difração de raio x do cromossomo tirada por Rosalind Franklin conseguiram entender como se dava a organização da molécula a partir da concepção do todo envolvido na nova explicação, enquanto não podemos afirmar que os mesmos tinham conhecimento do próprio processo de identificar o ADN. Isso mostra outro ponto importante sobre explicação e entendimento, que não necessariamente ambos se dão de forma propositiva, diferentemente do conhecimento (Zagzebski, 2001), como no exemplo ele se apresentou primeiramente pela percepção da imagem.

Com base no exposto aqui, então podemos propor um último argumento consequente, a ser:

4. O entendimento deve ser considerado como critério para a aceitação científica.

Essa conclusão pode ser defendida dado as limitações que uma associação ao conhecimento para a aceitação científica pode ter, seja no campo descritivo de como funciona esse processo na ciência seja na perspectiva normativa. Isso porquê ao focar na perspectiva que aceitação está associada ao Entendimento, há um reforço com pesquisas recentes sobre qual a função do ensino de ciências e a consequente formação de cientistas mais

$48 \quad$ Há o debate reducionista que define Entendimento a Conhecimento das causas. Não concordo com essa redução, dado que como apresentei aqui que Conhecimento é associado a conhecimento proposicional, além da questão da sorte epistêmica e há a possibilidade de haver conhecimento sem entendimento (vide caso apresentado). Para uma melhor discussão sobre esse tema, aconselho a revisão feita por Ferreira (2015) 
autônomos e responsáveis sobre o próprio processo de escolhas, seja no âmbito das ciências ou fora dela (Ferreira, 2015).

Indo nessa linha de pesquisa, podemos ver a importância do agente epistêmico de entender o conjunto de relações coerentes para então aceitar ou não dada teoria. Algo bastante próximo do que se compreende enquanto aceitação, que é o reconhecimento das razões que sustentam dado estado mental (Lehrer, 2000). Essa concepção de que a aceitação é derivada do entendimento daria caminho para a melhor compreensão de como se dá a relação do cientista seja com teorias novas sobre analise, seja sobre teorias rivais, dado que o entendimento dele sobre cada uma delas irá influenciar sua decisão de aceitar ou não as mesmas.

Assim, pode-se concluir que para se aceitar uma dada teoria o cientista, qua agente epistêmico, irá fazer com base naquela que ele melhor entende e isso resultara na escolha de uma teoria com maior valor, dado que é resultado de uma habilidade de um agente virtuoso. Pode parecer que ao dizer que o cientista opta por aceitar a teoria que mais entende seja uma grande obviedade, mas o que estamos dizendo aqui é que ao fazê-lo o cientista está do uso de sua habilidade, buscando ativamente fazer as relações entre as partes para entender o todo, e assim acessar o porquê daquelas partes estão ali e o que elas significam para o todo. Afinal, é isso que é entender, e para aceitar é necessário esse tipo de habilidade epistêmica.

\section{Referências:}

COHEN, Laurence J. An Essay on Belief and Acceptance. Oxford: Oxford University Press, 1992.

COHEN, Laurence J. Why Acceptance that P Does Not Entail Belief that P. In: ENGEL, Pascal. (Ed.). Beliving and Accepting. Philosophi ed. Dordrecht, Netherlands: Kluwer Academic, 2000. p. 55-65.

ELGIN, Catherine Z. Epistemic agency. Theory and Research in Education, v. 11, n. 2, p. 135-152, 2013.

ENGEL, Pascal. Beliving and Accepting. First ed. Dordrecht, Netherlands: Kluwer Acedemic, 2000.

FERREIRA, Tiago A. da S. Entendimento, Conhecimento e Autonomia: Virtudes Intelectuais e o Objetivo do Ensino de Ciências. [s.l.] UFBA, 2015. 
FRAASSEN, Bas van. A Imagem Científica. São Paulo, SP: Fundação Editora da UNESP, 2006.

GODFREY-SMITH, Peter. Theory and Reality - an introduction to the philosophy of science. Chicago, Illinois: The University of Chicago Press, 2003.

IVANOVA, Milena. Is There a Place for Epistemic Virtues in Theory Choice? In: FAIRWEATHER, Abrol. (Ed.). Virtue Epistemlogy Naturalized. Volume 336. Splinger, 2014. p. 207-227.

KUHN, Thomas. A estrutura das revoluções científicas. $5^{a}$ ed. São Paulo, SP: Editora Perspectiva, 1998.

LACEY, Hugh. Is Science Value Free? First ed. Lodon: Routledge, 1999.

LEHRER, Keith. Justification, Cohenrence and Knowledge. Erkenntnis, v. 50, p. 243-258, 1999.

LEHRER, Keith. Acceptance and Belief Revisited. In: ENGEL, Pascal. (Ed.). Beliving and Accepting. First ed. Dordrecht, Netherlands: Kluwer Acedemic, 2000. p. 209-220.

PRITCHARD, Duncan. Recent work on epistemic value. American Philosophical Quartely, v. 44, n. 2, p. 85-110, 2007.

RIGGS, Wayne D. Understanding "Virtue" and the Virtue of Understanging. In: ZAGZEBSKI, Linda.; DEPAUL, Michael. (Eds.). Intelectual Virtue. Paperback ed. Oxford: Oxford University Press, 2007. p. 203-226.

SAINSBURY, Mark. Fiction and Acceptance-Relative Truth, Belief and Assertion. In: Thuth in Fiction, 2011. p137-152.

ZAGZEBSKI, Linda. Recovering Understanding. In: STEUP, Mathias. (Ed.). Knowledge, Truth and Duty: Essays on Epistemic Justification, Responsability and Virtue. Oxford: Oxford University Press, 2001. p. 235-251. 